\title{
Descrição anatômica da articulação metacarpo-falângica em ovinos ${ }^{1}$
}

\author{
Pedro K.F.C. Bianchi²*, Edvaldo A.N. Martins ${ }^{3}$, Eloi Portugal ${ }^{3}$, José Roberto Kfoury \\ Júnior ${ }^{2}$ e Patrícia O. Gonçalez ${ }^{2}$
}

\begin{abstract}
Bianchi P.K.F.C., Martins E.A.N., Portugal E., Kfoury Júnior J.R. \& Gonçalez P.O. 2013. [Anatomical description of the metacarpal phalangeal joint in sheep.] Descrição anatômica da articulação metacarpo-falângica em ovinos. Pesquisa Veterinária Brasileira 33(Supl.1):15-19. Setor de Anatomia Animal, Faculdade de Veterinária, Universidade Camilo Castelo Branco, Av. Hilário da Silva Passos 950, Parque Universitário, Descalvado, SP 13690-970, Brazil. E-mail: pedro.bianchi@usp.br

The joints anatomical description highlights the standards for biomechanical conditions that must be considered in the selection of a particular animal species. Thus, the objective was to describe the metacarpal phalangeal joint in sheep, for its architecture and adjacent structures. For this purpose, we used ten adult sheep, Santa Ines breed, males and females, randomly chosen. The anatomical specimens for the study were collected by the disarticulation of the distal row of carpal and metacarpal bone of an sheep's forelimb. To observe the joint capsule, adjacent structures were overruled and their topographical relationships with such joint analyzed. The longitudinal measures of the joint capsule and the III-IV metacarpal bones, proximal phalanges III and IV proximal phalanx were taken. Through the results, it is concluded that the metacarpal phalangeal joint in sheep presents similar topographical relationships described for other ruminants, with connections at the proximal and recesses at the distal areas.
\end{abstract}

INDEX TERMS: Metacarpal phalangeal joint, sheep joint capsule, morphology, ruminants.

RESUMO.- A descrição anatômica articular evidencia os fatores padrões para as condições biomecânicas que devem ser consideradas na seleção do animal de uma determinada espécie. Dessa forma, objetivou-se descrever a articulação metacarpo-falângica na espécie ovina, em relação a sua arquitetura, considerando o volume, recessos, comunicações e relações no aspecto anatômico macroscópico. Para tanto, foram utilizados dez animais adultos da espécie ovina, raça Santa Inês, machos e fêmeas, escolhidos aleatoriamente. A cápsula articular teve uma dimensão média de 3,05 cm e uma capacidade volumétrica média de 3,99 ml; as comunicações ocorreram em um grau médio de expansão do Látex nas faces dorsal $(56,7 \%)$

\footnotetext{
${ }^{1}$ Recebido em 14 de setembro de 2013.

Aceito para publicação em 11 de novembro de 2013.

${ }^{2}$ Departamento de Cirurgia, Faculdade de Medicina Veterinária e Zootecnia (FMVZ), Universidade de São Paulo USP), Rua Prof. Orlando Marques de Paiva 87, São Paulo, SP 05508-270, Brasil. *Autor para correspondência: pedro.bianchi@usp.br.

${ }^{3}$ Faculdade de Medicina Veterinária, Universidade Camilo Castelo Branco (Unicastelo), Av. Hilário da Silva Passos 950, Parque Universitário, Descalvado , SP 13690-970, Brasil.
}

e palmar (53,3\%); as comunicações proximais nas faces dorsal, palmar e dorso-palmar apareceram em 100,0\% dos casos; os recessos estiveram presentes nas extremidades distais. Nesse contexto, conclui-se, que a articulação metacarpo-falângica de ovinos apresenta-se com relações topográficas semelhantes à descrita para os demais ruminantes, possuindo comunicações em sua porção proximal e recessos distalmente.

TERMOS DE INDEXAÇÃO: Articulação metacarpo-falângica, ovinos, cápsula articular, ruminante, morfologia.

\section{INTRODUÇÃo}

Em alguns países, inclusive no Brasil, o sistema de produção intensiva de ovinos vem crescendo significativamente, visto ser uma fonte de alimento de alta qualidade para o cosumo humano (IBGE 2005, Macrae \& Scott 1999).

As alterações no manejo, como o desmame precoce e as modificações nutricionais para obter uma engorda rápida, devem ser consideradas, pois podem culminar em problemas no desenvolvimento das estruturas corpóreas com possível envolvimento do aparelho locomotor. Sabe-se que 
uma das principais causas de patologias ósteo-articulares é o acelerado ganho de peso em animais jovens, devido à incapacidade das estruturas articulares suportarem o peso adquirido rapidamente (Alldred 1955).

Segundo Macrae e Scott (1999) e Berlingieri et al. (2011) , a claudicação causada por afecções que acometem as articulações é muito comum no Reino Unido, afetando o bem-estar dos animais, resultando em prejuízos para o sistema produtivo. Ainda, em carneiros jovens, a poliartrite infecciosa é a causa mais comum de doença articular, sendo encontrada principalmente no joelho (articulação fêmoro-tíbio-patelar), jarrete (articulação társica), carpo e boleto (articulação metacarpo-falângica).

Nas articulações metacarpo-falângicas (Schaller 1999), articulam-se as extremidades distais independentes dos ossos metacárpicos, na forma de uma tróclea, com a face articular da falange proximal e os dois sesamóides posicionados na face palmar (Nickel et al. 1986, König \& Liebich 2002, Dyce 2010, Duncan et al. (2013), e, segundo Getty (1986) e Banks (1992), há a existência de duas articulações, uma para cada dígito, com uma comunicação presente entre as duas partes palmares das duas cápsulas articulares.

No intuito de ampliar o conhecimento da região metacarpo-falângica, torna-se importante estudar aspectos anatômicos, considerando sua importância no contexto do acometimento em afecções articulares (Macrae \& Scott 1999).

Dessa forma, objetivou-se descrever a articulação metacarpo-falângica nos ovinos, considerando a capacidade volumétrica, recessos, comunicações e os limites da sua cápsula articular, obtendo-se dados que podem ser úteis na clínica médica e cirúrgica, mediante o melhor conhecimento anatômico da região.

\section{MATERIAL E MÉTODOS}

Utilizaram-se dez membros torácicos de ovinos adultos, raça Santa Inês, machos e fêmeas, escolhidos aleatoriamente, doados a partir de óbitos, ocorridos em criatórios da região de Descalvado, São Paulo. Após a obtenção das peças, estas foram congeladas para posterior preparo.

Na fase de preparo, as peças foram submetidas ao descongelamento natural por 24 horas. Após o descongelamento a articulação metacarpo-falângica foi acessada através da introdução dorsal de uma agulha, calibre 40x12G, realizando a injeção intra-articular de solução aquosa de Látex a 50\% corado com pigmento específico até a completa distensão da cápsula articular.

As peças injetadas com látex foram numeradas e conservadas por congelamento por três dias, visando o enrijecimento do produto, sendo descongeladas, após esse período, para dissecação da referida articulação.

Para uma melhor visualização e descrição dos dados, as comunicações foram organizadas segundo as regiões articulares de difusão do produto. Nas faces dorsal e palmar, foram consideradas as áreas de comunicação próximo-distal na região colateral do dedo IV (Pr-Dis IV), próximo-distal na região colateral do dedo III (Pr-Dis III), próximo-distal no espaço interaxial (Pr-Dis IntAx), interaxial na região proximal (IntAx Pr), interaxial na região distal (IntAx Dis) (Fig.1).

No eixo dorso-palmar, foram consideradas as áreas de comunicação proximal na região colateral do dedo III (Pr III), proximal

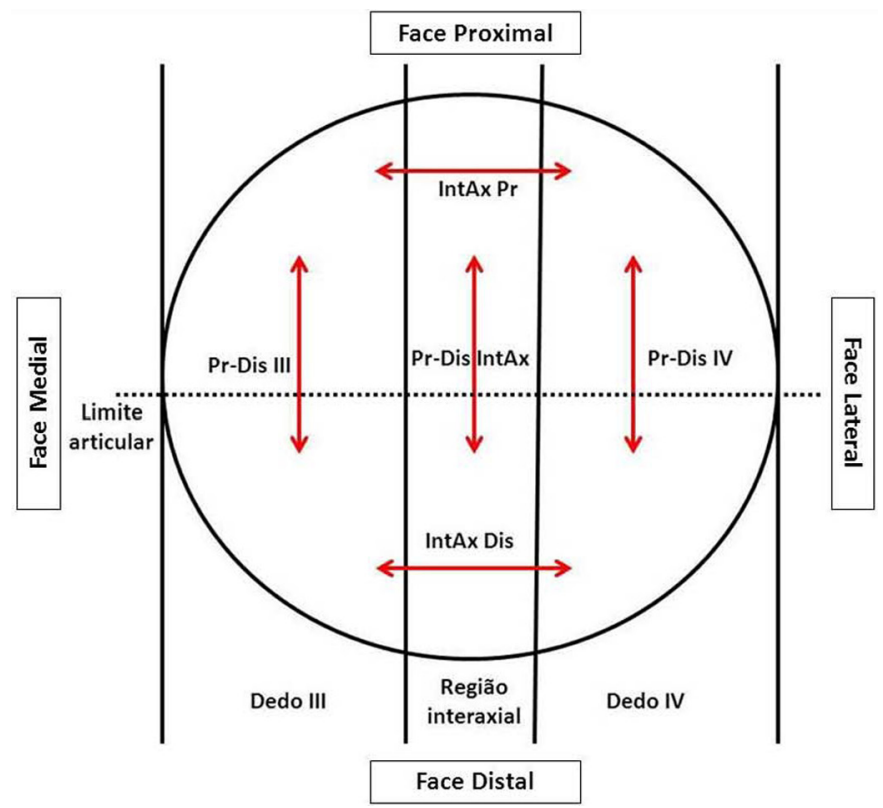

Fig.1. Esquema para observação das áreas de comunicação da articulação metacarpo-falângica de ovinos nas faces dorsal e palmar.

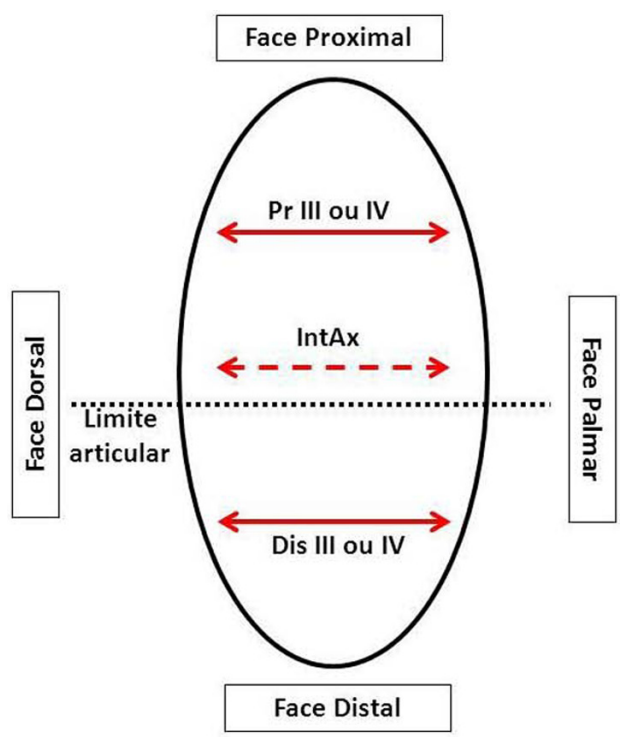

Fig.2. Esquema para observação das áreas de comunicação da articulação metacarpo-falângica de ovinos no eixo dorso-palmar.

na região colateral do dedo IV (Pr IV), distal na região colateral do dedo III (Dis III), distal na região colateral do dedo IV (Dis IV) e Interaxial (IntAx) (Fig.2).

Após a dissecação articular, os ossos envolvidos (metacárpicos III-IV, falange proximal III e falange proximal IV) foram mensurados longitudinalmente para referência comparativa ao tamanho da cápsula articular.

Da mesma forma, os recessos presentes também foram observados de acordo com as regiões dorso-proximal (Dr Pr), dorso-distal (Dr Dis), palmo-proximal (Pal Pr) e palmo-distal (Pal Dis).

O grau de comunicação foi estipulado em nível um, quando apenas um ducto foi preenchido; dois, quando metade da região permitia a passagem do látex; e três, quando o látex se encontrava em toda a região de comunicação. Quando o látex não preencheu a região de observação, a comunicação foi considerada ausente. 
Os dados obtidos foram analisados por estatística descritiva e confrontados com a literatura, assim como foram tomadas fotografias para comprovação e ilustração das informações.

\section{RESULTADOS}

Após a dissecção das estruturas adjacentes à articulação dos ovinos foi possível visualizar e mensurar a cápsula articular. Essa se fixou ao redor das margens articulares (ossos metacarpo III-IV e falanges proximal III e IV), havendo uma pequena variação na distância entre os pontos de fixação.

Em todas as peças o volume de Látex injetado para a expansão completa da cápsula articular, bem como as medidas da cápsula articular e dos segmentos ósseos envolvidos na articulação, foram mensurados para comparação.

A correlação entre os fatores volume e mensuração de cápsula articular (Quadro 1) foi 0,10 (fraca). Ao se correlacionar a mensuração longitudinal da cápsula articular às dimensões longitudinais do metacarpo III-IV e das falanges proximal III e IV, obteve-se índices 0,25, -0,04 e -0,03, respectivamente (fracos). Porém, o comportamento do volume articular perante as dimensões longitudinais do metacarpo III-IV e das falanges proximal III e IV apresentou correlação moderada $(0,39,0,65$ e 0,5 , respectivamente).

Após o completo rebatimento da cápsula articular foi possível à visualização intra-articular satisfatória do comportamento da cavidade articular, devido ao molde de Lá-

\section{Quadro 1. Mensurações do volume (ml) de expansão da cápsula articular e das dimensões longitudinais (cm) das estruturas envolvidas na formação da articulação metacarpo-falângica em ovinos}

\begin{tabular}{lccc}
\hline Observações & Média & Desvio Padrão & Coeficiente de Variação \\
\hline Volume articular & 3,99 & 0,53 & $13,44 \%$ \\
Cápsula articular & 3,05 & 0,62 & $20,57 \%$ \\
Metacarpo III-IV & 12,29 & 1,66 & $13,54 \%$ \\
Falange proximal III & 3,12 & 0,20 & $6,55 \%$ \\
Falange proximal IV & 3,15 & 0,24 & $7,81 \%$
\end{tabular}

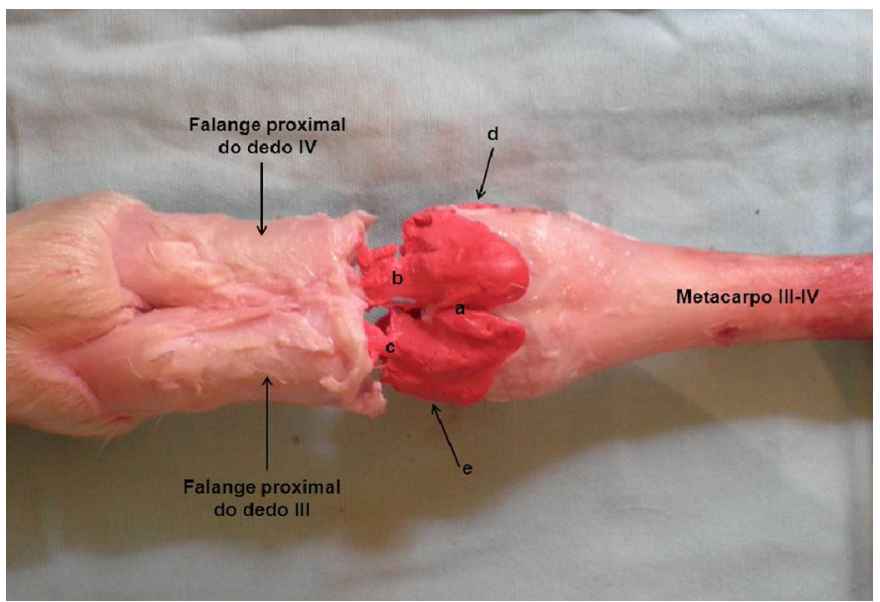

Fig.3. Vista dorsal da articulação metacarpo-falângica na espécie ovina, evidenciando o preenchimento da cavidade articular por látex corado. a. comunicação inter-axial proximal grau 3. b. comunicão proximo distal IV grau 2. c. comunicação proximo-distal III grau 2. d. comunicação dorso-palmar proximal IV grau 1. e. comunicação dorso-palmar proximal III grau 1.

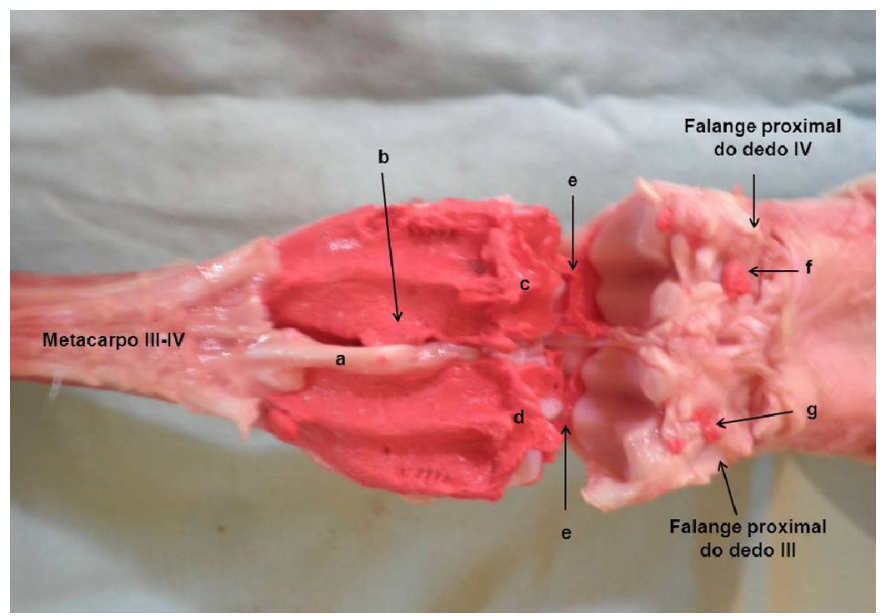

Fig.4. Vista palmar da articulação metacarpo-falângica na espécie ovina, evidenciando o preenchimento da cavidade articular por látex corado. a. lâmina axial do m. interósseo para o tendão dos mm. extensores. b. comunicão inter-axial proximal grau 3. c. comunicação proximo-distal IV grau 2. d. comunicação proximo-distal III grau 2. e. comunicação dorso-palmar inter-axial grau 1. f. recesso palmo-distal na região do dedo IV. g. recesso palmo-distal na região do dedo III.

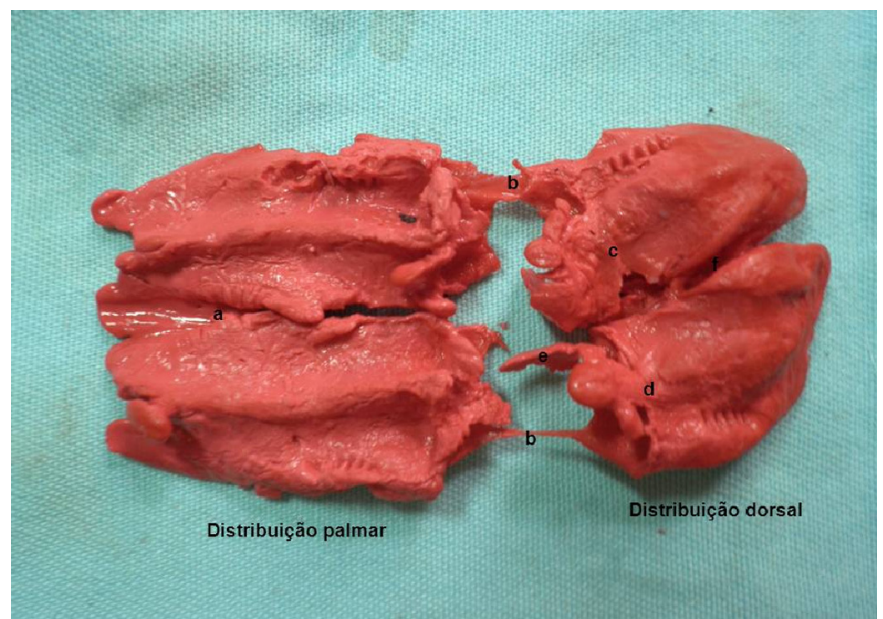

Fig.5. Molde de látex corado da articulação metacarpo-falângica na espécie ovina. a. comunicação palmar inter-axial proximal grau 3. b. comunicão dorso-palmar inter-axial grau 1. c. comunicação dorsal proximo-distal III grau 2. d. comunicação dorsal proximo-distal IV grau 2. e. recesso dors-distal na região do dedo IV. f. comunicação dorsal inter-axial proximal grau 3.

tex formado. 0 grau de comunicação entre as diferentes partes da cápsula foi considerado ausente ou avaliado em uma escala de um a três, de acordo com a quantidade de Látex difundido entre as partes no interior da articulação metacarpo-falângica (Fig.3-5).

As comunicações articulares foram observadas de acordo com as regiões pré-determinadas e os resultados referentes a essas estão dispostos nos Quadros 2 a 4.

Foram observados recessos nas regiões dorso-distal e palmo-distal, ambas nos dedos III e IV. Porém em 100\% das dissecações não foram observados recessos nas regiões dorso-proximal e palmo-proximal. 
Quadro 2. Comunicações regionais da cavidade articular observadas na face dorsal da articulação metacarpofalângica em ovinos

\begin{tabular}{lcccc}
\hline Região & \multicolumn{5}{c}{ Grau } \\
\cline { 2 - 5 } & 1 & 2 & 3 & Total \\
\cline { 2 - 5 } & $\mathrm{N}$ & $\mathrm{N}$ & $\mathrm{N}$ & $\mathrm{N}$ \\
\hline Pr-Dis III & 5 & 5 & 0 & 10 \\
Pr-Dis IV & 0 & 10 & 0 & 10 \\
Pr-Dis IntAx & 0 & 0 & 0 & 0 \\
IntAx Pr & 0 & 2 & 8 & 10 \\
IntAx Dis & 0 & 0 & 0 & 0 \\
TOTAL & 5 & 17 & 8 & 30
\end{tabular}

Quadro 3. Comunicações regionais da cavidade articular observadas na face palmar da articulação metacarpofalângica em ovinos

\begin{tabular}{lcccc}
\hline Região & \multicolumn{5}{c}{ Grau } \\
\cline { 2 - 5 } & 1 & 2 & 3 & Total \\
\cline { 2 - 5 } & $\mathrm{N}$ & $\mathrm{N}$ & $\mathrm{N}$ & $\mathrm{N}$ \\
\hline Pr-Dis III & 6 & 4 & 0 & 10 \\
Pr-Dis IV & 3 & 7 & 0 & 10 \\
Pr-Dis IntAx & 0 & 0 & 0 & 0 \\
IntAx Pr & 0 & 5 & 5 & 10 \\
IntAx Dis & 0 & 0 & 0 & 0 \\
TOTAL & 9 & 16 & 5 & 30
\end{tabular}

Quadro 4. Comunicações regionais da cavidade articular observadas no eixo dorso-palmar da articulação metacarpofalângica em ovinos

\begin{tabular}{lcccc}
\hline Região & \multicolumn{5}{c}{ Grau } \\
\cline { 2 - 5 } & 1 & 2 & 3 & Total \\
\cline { 2 - 5 } & $\mathrm{N}$ & $\mathrm{N}$ & $\mathrm{N}$ & $\mathrm{N}$ \\
\hline Pr III & 3 & 7 & 0 & 10 \\
Pr IV & 3 & 7 & 0 & 10 \\
Dis III & 0 & 0 & 0 & 0 \\
Dis IV & 0 & 0 & 0 & 0 \\
IntAx & 4 & 6 & 0 & 10 \\
TOTAL & 10 & 20 & 0 & 30
\end{tabular}

\section{DISCUSSÃO}

A variação observada na distância entre os pontos de fixação da cápsula articular pode ser um reflexo da rigidez e resistência assumida pela cápsula devido à exigência das forças aplicadas sobre o local como discorrem Alldred (1955) e König \& Liebich (2002).

Os dados coletados apresentaram baixos coeficientes de variação, o que demonstra uma pequena importância na dispersão dos dados em torno da média dada pelo desvio padrão. A correlação fraca vista ao se equiparar o volume e a mensuração de cápsula articular e esta última com o comprimento dos ossos envolvidos, indica independência entre os fatores. 0 aumento da correlação entre o volume articular e às dimensões longitudinais ósseas pode sugerir que o fator que altera a capacidade volumétrica da articulação está relacionado com a requisição de movimento e força estática óssea.

A face dorsal apresentou comunicações em todas as peças estudadas em três das cinco regiões analisadas, sendo que as regiões próximo-distal, inter-axial e inter-axial distal não apresentaram correspondência. A comunicação próximo-distal na região colateral dos dedos III e IV foi vis- ta em todas as dissecações, diferenciando-se em grau 1 ou 2. 0 espaço inter-axial proximal apresentou-se como uma comunicação relevante, já que foi a única região a obter grau 3 (80,0\% das peças).

O comportamento da face palmar foi semelhante ao notado na face dorsal. A ausência de correspondência distal também foi relatada, o que demonstra que, independente da face, essas comunicações são limitadas. Tal fato pode ser explicado pela maior presença de estruturas que se fixam nessa porção, provocando maior tensão na cápsula com consequente diminuição de espaço. 0 espaço inter-axial proximal também foi o único a apresentar grau 3, porém em um menor número de casos $(50,0 \%)$.

De uma maneira geral, a face dorsal e palmar apresentaram em maior número o grau de comunicação 2 (56,7\% e $53,3 \%$, respectivamente).

Autores como Getty (1986), Nickel et al. (1986) e König \& Liebich (2002) comentam que na articulação metacarpo-falângica há dois processos articulares, sendo um para cada dígito, havendo uma comunicação na região palmar entre as duas cápsulas articulares. Somente Schwarze (1970) não especifica que esta ocorre na região palmar. Porém, neste estudo, observou-se também, em 100\% dos casos, interação em pontos regionais diferentes na face dorsal, o que não foi citado pela literatura consultada.

Nas comunicações do eixo dorso-palmar, foi observado proximalmente, tanto para a região colateral do dedo III como na do dedo IV, o grau 2 em 70,0 \% e de grau $1 \mathrm{em}$ $30,0 \%$ dos casos. Distalmente, não houve sinais de difusão do produto, concluindo-se uma ausência de comunicação. 0 grau de comunicação inter-axial foi representativo, sendo $60 \%$ grau 2 e $40 \%$ grau 1 ; novamente, o grau de comunicação mais frequente foi o $2(66,7 \%)$.

Notou-se a presença do Látex em regiões um pouco distante da articulação, confirmando a formação de saculações. Sugere-se que estas possam ajudar na movimentação dos tendões (Getty 1986), embora sejam bastante discretas nesses animais.

Os recessos foram descritos somente nas regiões articulares distais. Fato interessante, ao se associar que as comunicações distais não ocorreram nas faces dorsal, palmar e dorso-palmar, podendo sugerir que a limitada cavidade nessa região impossibilita a comunicação entre as regiões, mas permite a formação de recessos.

Contudo, os resultados desta pesquisa mostraram que as comunicações proximais na face dorsal, palmar e dorso- palmar aparecem em todos os casos, porém a difusão do látex não foi observada entre as regiões distais.

Considerando a cápsula articular, sua dimensão média foi de $3,05 \mathrm{~cm}$ e sua capacidade volumétrica média foi de $3,99 \mathrm{ml}$. No entanto, as dimensões médias longitudinais dos ossos metacarpo III-IV e falanges proximais III e IV tiveram correlação fraca ou moderada com a mensuração longitudinal da cápsula articular e seu volume, respectivamente.

Ainda, considerando os recessos articulares, vale ressaltar que estes estiveram presentes nas extremidades distais, porém não foram observados nas porções proximais. 


\section{REFERÊNCIAS}

Alldred A.J. 1955. Rupture of the collateral ligament of the metacarpophalangeal joint of the thumb. J. Bone Joint Surg. 3:443-445.

Banks W.J. 1992. Histologia Veterinária Aplicada. 2aㅡ ed. Manole, São Paulo. $655 p$.

Berlingieri M.A., Catellan W.J., Baraldi Artoni M.S. 2011. Aspectos morfológicos da face flexora dos dígitos de novilhas mestiças da raça nelore. Ars Vet. 27:66-72.

Duncan S.J., Singer R.E., Devaney J., Oultram W.H., Walby J.A., Lester R.B., Williams J.H. 2013. The radiographic anatomy of the normal ovine digit, the metacarpophalangeal and metatarsophalangeal joints. Vet. Res. Commun., Liverpool, 37:51-57.

Dyce K.M., Sack W.O. \& Wensing C.J.G. 2004. Tratado de Anatomia Veterinária. 3a ed. Elsevier, Rio de Janeiro. 834p.

Getty R. 1986. Anatomia dos Animais Domésticos. 5a ed. Guanabara Koogan, Rio de Janeiro. 2000p.
IBGE 2005. Pesquisa Pecuária Brasileira. Disponível em <http://www. ibge.gov.br/home/estatística/economia,ppm/tabela1ppm_2001.shtm> Acesso em 10 jul. 2011.

König E.H. \& Liebich H.G. 2002. Anatomia dos Animais Domésticos: aparelho locomotor. Artmed, Porto Alegre. 787p.

Macrae A.I. \& Scott P.R. 1999. The normal ultrasonographic appearance of ovine joints, and the uses of arthrosonography in the evaluation of chronic ovine joint disease. Vet. J. 158:135-143.

Nickel R., Schummer A., Seiferle E., Wilkens H., Wille K-H. \& Frewein J. 1986. The Anatomy of the Domestic Animals: the locomotor system of the domestic mammals. Verlag Paul Parey, Berlin. 499p.

Schaller 0. 1999. Nomenclatura Anatômica Veterinária Ilustrada. Manole, São Paulo. 614p.

Schwarze E. 1970. Compendio de Anatomia Veterinária: introdución a la anatomia veterinária - aparato locomotor. Acribia, Zaragoza. 319p. 